\title{
Heart-valve Sounds Obtained with a Laser Doppler Vibrometer
}

\author{
Johannes J Struijk, Kim Munck, Bolette D Hansen, Nina Jacobsen, Louise P Pilgaard, \\ Kasper Sørensen, Samuel E Schmidt
}

\author{
Aalborg University, Aalborg, Denmark
}

\begin{abstract}
The present work investigates the use of a Laser Doppler Vibrometer (LDV) to record valve sounds and characterizes those recordings.

An LDV (Polytec PDV-100) was used to measure the normal component of the velocity of the chest wall at 30 points (grid of $6 \times 5$ points) in each of seven subjects. The recorded signals were analyzed in the frequency domain and filtered to obtain the S1 and S2 heart sounds. From all grid points a center of energy (CoE) was calculated for the signal energy, separately for the S1 and S2 sounds.

The LDV signal reached a noise floor for a frequency of $>80 \mathrm{~Hz}$, whereas $99 \%$ of the signal energy was below 80 $\mathrm{Hz}$. The signal to noise ratio was approximately $24 \mathrm{~dB}$ if measured directly on the (caucasian) skin, and up to $45 \mathrm{~dB}$ if special markers were used. There was little difference in signal amplitudes between measurement points on the ribs as compared with the intercostal spaces. Amplitudes of the valve sounds were below $1 \mathrm{~mm} / \mathrm{s}$. The mean centers of energy were to the left of the sternum, with the CoE of the $S 2$ sound about $11 \mathrm{~mm}$ superior to the CoE of the S1 sound on average.

The LDV is an interesting new, non-contact, modality for the recording of heart sounds with excellent signal quality and reproducibility.
\end{abstract}

\section{Introduction}

Several modalities for recording of heart sounds have been used since the late 1800s. Microphones and accelerometers have been the obvious choices since the beginning of the $20^{\text {th }}$ century, showing that a wealth of information about cardiac movement is available, also outside the audible frequency range, as well as about the audible valve sounds and murmurs. A relatively new method in cardiac "auscultation" is the use of laser Doppler Vibrometry (LDV), which is a contactless method which can be used to measure skin movement [1-4].

In the current paper we focus on the high frequency content (valve sounds) of the LDV signal obtained from the chest, whereas a companion paper is focused on the low-frequency content (movement of the cardiac wall).

\section{Methods}

Seven healthy caucasian volunteers (4 male / 3 female, age 22-52 years) were enrolled in the study. The subjects were in a supine position and were instrumented with a 3lead ECG, and a respiration belt (figure 1). Grid positions for the LDV recordings were marked on the skin with the help of a projector. The positions (6 rows and 5 columns) are shown in figure 2. The LDV was a Polytec PDV-100 and it was positioned in a vertical position as shown in fig. 1. Data acquisition was performed with an iWorx RA-834 and included the ECG, the respiration signal, the LDV and the signal of an accelerometer that was placed on the moveable arm bearing the LDV, to monitor excessive vibration of the equipment.

The LDV signal was filtered with the built-in highpass filter $(1 \mathrm{~Hz})$ and lowpass filter $(1 \mathrm{kHz})$, before being sampled at $10 \mathrm{kHz}$. The output of the LDV was the velocity with a resolution of $5 \mathrm{~mm} / \mathrm{s} / \mathrm{V}$.

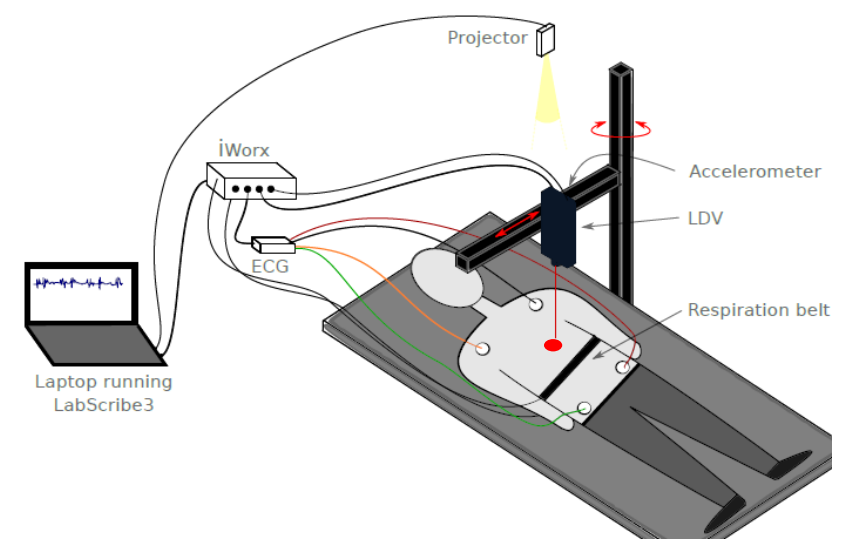

Figure 1. Overview of the measurement setup. 
The LDV signal contained a large number of dropouts (between 0 and 20 per second with a duration of $<4 \mathrm{~ms}$ ) which were detected automatically and removed by interpolation of the signal across the dropouts.

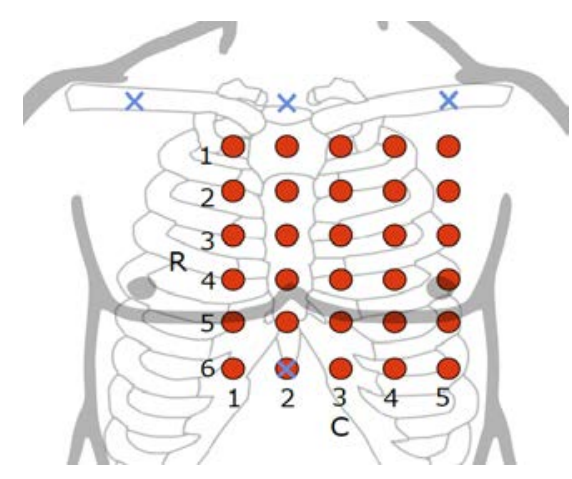

Figure 2. Red dots: Recording positions of the LDV divided in 6 rows and 5 columns.

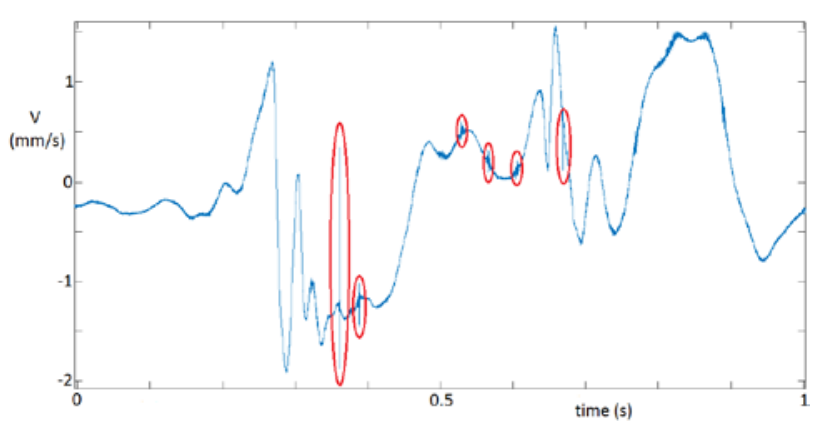

Figure 3 - One second of raw LDV signal (velocity) with bandwidth of $1 \mathrm{~Hz}$ to $1 \mathrm{kHz}$. The red markers indicate dropouts appearing as short peaks.

The signals were subsequently bandpass filtered (20-80 $\mathrm{Hz}$ ) to isolate the valve sounds, using an FIR filter (1024 filter taps) to avoid phase distortion. See figure 4.

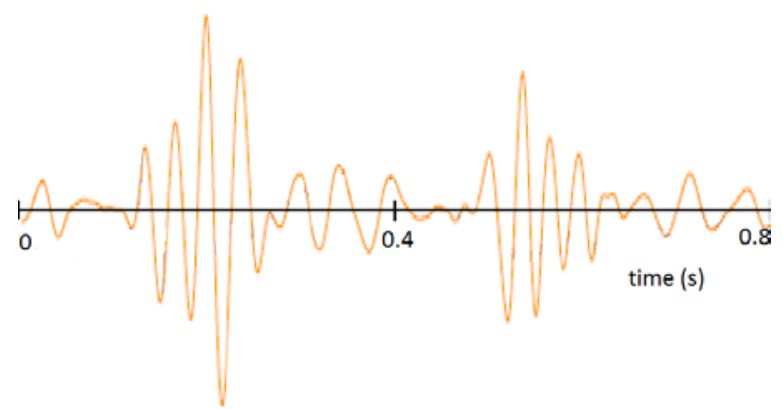

Figure 4 - S1 and S2 sounds in a 20-80 Hz bandwidth. The peak velocity in this example is $0.13 \mathrm{~mm} / \mathrm{s}$.
S1 and S2 sounds were detected and their signal energies were calculated. From the signal energies, for each subject two Centers of Energy (CoE) were calculated, one for S1 and one for S2.

\section{Results}

The LDV signal reached a noise floor at a frequency of $>80 \mathrm{~Hz}$, with $99 \%$ of the signal energy below $80 \mathrm{~Hz}$. The signal to noise ratio was approximately $24 \mathrm{~dB}$ if measured directly on the (caucasian) skin, and up to $45 \mathrm{~dB}$ if reflecting markers were used. There was little difference in signal amplitudes between measurement points on the ribs as compared with the intercostal spaces. Amplitudes of the valve sounds were below $1 \mathrm{~mm} / \mathrm{s}$. The mean centers of energy were to the left of the sternum, with the CoE of the $\mathrm{S} 2$ sound about $11 \mathrm{~mm}$ superior to the CoE of the $\mathrm{S} 1$ sound on average.
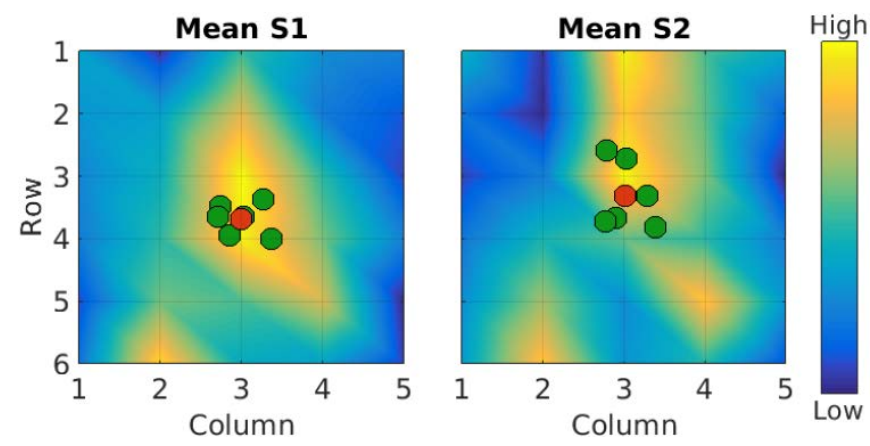

Figure 5 - Energies of the S1 and S2 sounds related to the grid positions. Colors blue to yellow indicate low to high energies. Green dots: $\mathrm{CoE}$ for each of the subjects. Red dots: Average CoE for all subjects.

\section{Discussion}

As compared with microphone recordings, the LDV has a rather low frequency band of up to $80 \mathrm{~Hz}$ beyond which the noise becomes a limiting factor. The use of reflecting markers on the skin improves the SNR significantly, but we have not yet investigated this in a systematic way. The use of reflectors also reduces the number of dropouts significantly. However, we have shown that the valvular sounds S1 and S2 can be measured with a high signal quality in agreement with the results of Koegelenberg et al. [3].

The strongest signals were obtained with the LDV position at the left sternal border approximately at the $3^{\text {rd }}$ and $4^{\text {th }}$ intercostal spaces, where S2 also radiated upwards (superior) and S1 somewhat in an apical direction. The 
center of energy for the S2 sound was $11 \mathrm{~mm}$ superior as compared with $\mathrm{S} 1$, which may be related to the relative anatomical positions of the mitral and aortic valves, but which is mostly caused by the rostral radiation of the S2 sound.

Laser Doppler cardiography is thus a very interesting alternative for the use of microphones, since the sound is measured in a contactless way, thus avoiding the introduction of skin loading and signal distortion.

The bulky and expensive equipment may be prohibitive for a widespread use of the LDV today, but new advances towards small solid state LDVs [5] promise a bright future for laser Doppler vibrocardiography.

\section{Acknowledgements}

This work was made possible by a grant from "Det Obelske Familiefond”.

\section{References}

[1] Tabatabai H, Oliver DE, Rohrbaugh JW, Papadopoulos C. Novel applications of laser Doppler vibration measurements to medical imaging. Sens Imaging 2013;14:13-20.

[2] Morbiducci U, Scalise L, De Melis M, Grigioni M (2006). Optical vibrocardiography: a novel tool for optical monitoring of cardiac activity. Ann Biomed Engng 2006;35:45-58.

[3] Schuurman T, Rixen DJ, Swenne CA, Hinnen JW. Feasibility of laser Doppler vibrometry as potential diagnostic tool for patients with abdominal aortic aneurysms. J Biomech 2013;46:1113-1120.

[4] Koegelenberg S, Scheffer C, Blanckenberg MM, Doubell AF. Application of laser Doppler Vibrometry for human heart auscultation. IEEE EMBS Conf Proc 2014;4479-4482.

[5] Li Y, Segers P, Dirckx J, Baets R. On-chip laser Doppler vibrometer for arterial pulse-wave velocity measurement. Biomed Opt Express 2013;4:1229-1235.
Address for correspondence.

Johannes Jan Struijk

Dept. Health Science and Technology

Aalborg University

Fredrik Bajers Vej 7C1-205

9220 Aalborg

Denmark

jis@hst.aau.dk 
